\title{
Trendelenburg chest optimization prolongs spontaneous breathing trials in ventilator-dependent patients with low cervical spinal cord injury
}

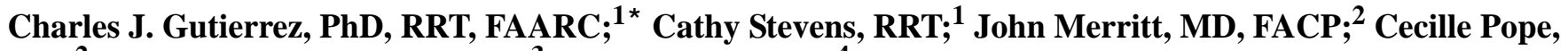 \\ MD; ${ }^{2}$ Mihaela Tanasescu, MD, ScD; ${ }^{3}$ Glenn Curtiss, PhD $^{4}$ \\ ${ }_{1}^{1}$ Neurorespiratory Care Program, Department of Respiratory Care \& Diagnostic Services, and ${ }^{2}$ Spinal Cord Injury/ \\ Disorders Service, Bilirakis Spinal Cord Injury Center of Excellence (SCICoE), James A. Haley Veterans Hospital, \\ Tampa, FL; ${ }^{3}$ Touro University International, College of Health Sciences, Cypress, CA; ${ }^{4}$ Department of Neuropsychol- \\ ogy, Bilirakis SCICoE, James A. Haley Veterans Hospital, Tampa, FL
}

\begin{abstract}
Chest optimization, an evidence-based protocolguided multimodal chest physiotherapy consisting of body positioning, sputum mobilization, bronchodilation, and lung hyperinflation, may be routinely administered to ventilatordependent patients with low cervical spinal cord injury (CSCI) for improving pulmonary functional outcomes that facilitate weaning from mechanical ventilation. We undertook this study to determine whether position-specific chest optimization was associated with changes in spontaneous breathing trial (SBT) duration. Cardiac output (CO), alveolar minute volume (MValv), carbon dioxide elimination $\left(\mathrm{VCO}_{2}\right)$, and static chest compliance (Cst) were measured during chest optimization; then MValv and rapid shallow breathing index (RSBI) were measured during SBT. Study participants $(N=12)$ were clinically stable ventilator-dependent patients with low CSCI. Trendelenburg chest optimization (TCO) was associated with significant increases in SBT $(p<0.001)$, CO $(p<0.001)$, MValv $(p<0.003), \mathrm{VCO}_{2}(p<0.001)$, and Cst $(p<0.002)$. SBT following TCO was associated with significant increases in MValv $(p<0.03)$ and RSBI $(p<0.002)$. These preliminary findings suggest the importance of proper recumbent body positioning during evidence-based, protocol-guided multimodal chest physiotherapy for ventilator-dependent patients with low CSCI.
\end{abstract}

Key words: alveolar minute volume, body positioning, bronchodilation, carbon dioxide elimination, cardiac output, cervical spinal cord injury, chest optimization, chest physiotherapy, evidence-based, lung hyperinflation, multimodal, noninvasive cardiac output monitoring, rapid shallow breathing index, rehabilitation, spontaneous breathing trial, sputum mobilization, static chest compliance, supine, tetraplegia, Trendelenburg.

Abbreviations: ASIA = American Spinal Injury Association, $\mathrm{C}=$ cervical, $\mathrm{CO}=$ cardiac output, $\mathrm{CO}_{2}=$ carbon dioxide, $\mathrm{CPAP}=$ continuous positive airway pressure, $\mathrm{CSCI}=$ cervical spinal cord injury, CSCM = Consortium for Spinal Cord Medicine, Cst $=$ static chest compliance, $\mathrm{FIO}_{2}=$ fractional inspired oxygen concentration, FRC = functional residual capacity, GIN = gas injection nebulizer, $\mathrm{H}_{2} \mathrm{O}=$ dihydrogen monoxide (water), $\mathrm{HR}=$ heart rate, IBW = ideal body weight, IRB = institutional review board, MIE = mechanical insufflator/exsufflator, MValv = alveolar minute volume, $\mathrm{NICO}=$ noninvasive $\mathrm{CO}$, $\mathrm{RSBI}=$ rapid shallow breathing index, $\mathrm{SBT}=$ spontaneous breathing trial, $\mathrm{SCI}=$ spinal cord injury, $\mathrm{SCO}=$ supine chest optimization, $\mathrm{SpO}_{2}=$ pulse oximetric saturation, $\mathrm{SVN}=$ small volume nebulizer, $\mathrm{TCO}=$ Trendelenburg chest optimization, $\mathrm{VA}=$ Department of Veterans Affairs, $\mathrm{VC}=$ vital capacity, $\mathrm{VCO}_{2}=\mathrm{CO}_{2}$ elimination, $\mathrm{WBC}=$ white blood cell.

* Address all correspondence to Charles J. Gutierrez, PhD, RRT, FAARC; Neurorespiratory Care Program, Bilirakis SCI Center (128C), James A. Haley Veterans Hospital, 13000 Bruce B. Downs Blvd, Tampa, FL 33612; 813-6107329. Email: charles.gutierrez@va.gov

DOI:10.1682/JRRD.2009.07.0099 


\section{INTRODUCTION}

Low tetraplegic injuries involve cervical (C) spinal cord levels C3 through C7 and are associated with greater preservation of ventilatory function than those with high tetraplegic injuries. Ventilator-dependent patients with cervical spinal cord injury (CSCI) are prone to multiple pulmonary complications that include atelectasis, mucus plugging, and pneumonia. Pulmonary complications are directly related to level of injury and completeness of neuronal dysfunction. Patients with complete injuries exhibit an increase in frequency and severity of ventilatory difficulties [1]. Pneumonia is the principal pulmonary complication in those with high-level cervical injuries, while atelectasis is the most common complication in individuals with low-level cervical injuries [2]. Main muscles of inspiration include diaphragm, which contributes 65 to 75 percent of tidal volume during normal ventilation [3-5], as well as external intercostals and accessory muscles, which together account for the remaining percentage of ventilation. Main muscles of expiration are internal intercostals, rectus abdominis, transversus abdominis, internal and external oblique, and pectoralis major. Adequate function of abdominal muscles supports adequate minute ventilation and is important in the ability to shear secretions adequately during a cough reflex [6]. When cough is ineffective, therapeutic modalities are used to disencumber the lungs by clearing secretions from the smallest, most peripheral airways [7] to improve pulmonary function and improve prospects for weaning.

Patients hospitalized with CSCI exhibit an 80 percent mortality rate due to pulmonary complications, with pneumonia the cause of death in 50 percent of cases [1,810]. Persistent, systematic, and intensive respiratory therapy for those with spinal cord injury (SCI) has improved pulmonary functional outcomes [11-13], which may therefore improve prospects for weaning from mechanical ventilation. Prompt, aggressive management of pulmonary complications by respiratory therapists dedicated to a specialized SCI rehabilitation unit has been shown to mitigate pulmonary complications effectively, including pneumonia, and reduce the likelihood of requiring bronchoscopic intervention [11-12]. Prevention of pulmonary complications should begin immediately after SCI [11] and should continue through subacute and chronic phases [14]. Preliminary experience of patients with low tetraplegia at our specialized clinical facility suggests that an evidence-based protocol-guided neuro- respiratory rehabilitative approach improves efficiency and effectiveness of liberation from mechanical ventilation [15-16]. The use of evidence-based protocol-guided neurorespiratory rehabilitation will assume greater importance given that by 2025, the population of the United States is projected to grow by 20 percent and the disease burden is expected to shift from acute disorders to chronic disease and disability [14].

Chest optimization is systematic administration of evidence-based protocol-guided (see Appendix, available online only) multimodal chest physiotherapy to ventilatordependent patients with low tetraplegia. It enhances pulmonary functional outcomes in preparation for respiratory rehabilitation intended to expedite weaning patients from mechanical ventilation (Figure 1). While primarily intended as a prophylaxis for pulmonary complications, chest optimization may also be used therapeutically. The term suggests the importance of routinely optimizing chest and lung function by way of gravitational, mechanical, vibrational, and pharmacological means to improve resistance and endurance training outcomes that may facilitate weaning. Aggressive multimodal chest physiotherapy

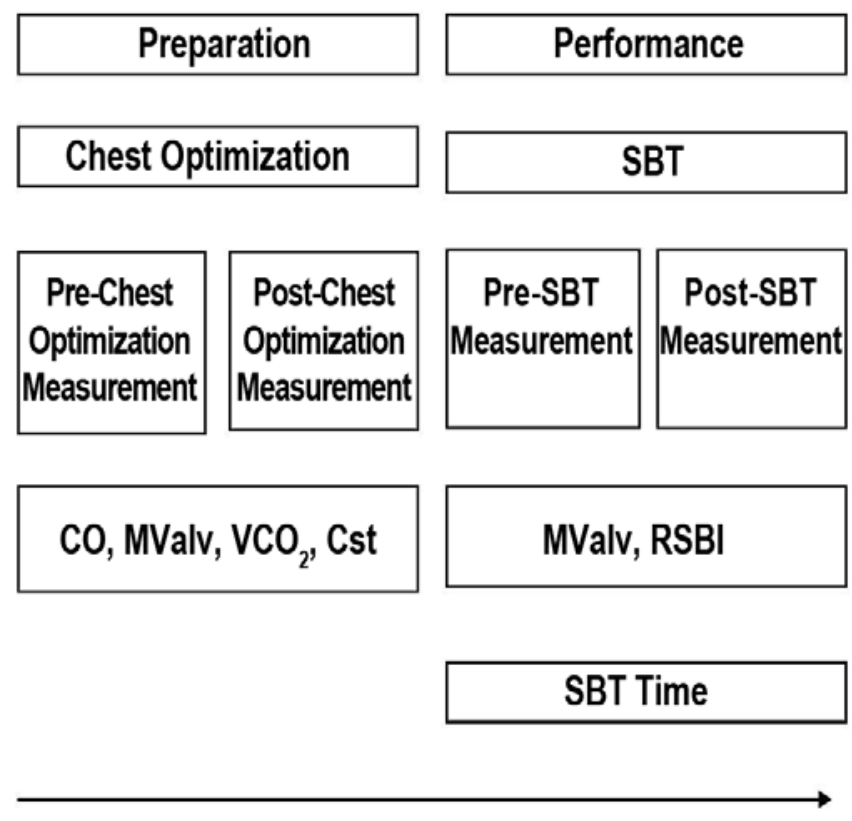

Timeline

Figure 1.

Measurement timeline of chest optimization and spontaneous breathing trial $(\mathrm{SBT}) . \mathrm{CO}=$ cardiac output, $\mathrm{Cst}=$ static chest compliance, MValv = alveolar minute volume, RSBI = rapid shallow breathing index, $\mathrm{VCO}_{2}$ = carbon dioxide elimination. 
may mitigate or prevent pulmonary complications [14]. Based on extensive reviews of the scientific literature, Dean concluded that body positioning should be a firstline intervention for patients with cardiopulmonary dysfunctions [17]. Hence, the current study examined the role of recumbent body positioning during chest optimization on duration of spontaneous breathing trials (SBTs).

\section{METHODS}

\section{Subjects}

The current study was a double-blind, randomized clinical intervention trial with crossover experimental design. Patients $(N=12)$ were randomly assigned to supine chest optimization (SCO) or Trendelenburg chest optimization (TCO) (head-down position). Generally, improved pulmonary functional outcomes last 3 to 4 hours following multimodal chest physiotherapy. Hence, a 12hour washout period between interventions was used to mitigate carryover effects [6]. Subjects were Active Duty or retired male military veterans, aged 24 to 74, with ventilator dependence due to ventilatory failure associated with subacute or chronic traumatic CSCI. All were admitted to an acute rehabilitation ventilator unit in a major regional referral SCI center to undergo protocolguided neurorespiratory rehabilitation and ventilator weaning. While all subjects received nonsequential components of chest optimization in the past, they were medically naïve to chest optimization as a bundled, routinely administered clinical intervention consisting of four modalities that included body positioning, sputum mobilization, bronchodilation, and lung hyperinflation. All participants were medically stable, well-nourished, and free from significant atelectasis, mucus plugging, or pneumonia. Lack of pulmonary complications may have been due to the routine use of SCI-specific tidal volumes (15 mL/kg ideal body weight [IBW]) and mechanical insufflation/exsufflation [18]. Chest X-rays were ordered on an as-needed basis. Patients were on continuous pulse oximetry of 21 percent fractional inspired oxygen concentration $\left(\mathrm{FIO}_{2}\right)$, which enabled quick detection of hypoxemia and/or hypercarbia secondary to atelectasis.

Subjects ingested food by month and/or feeding tube at the same time each day and were closely monitored by speech pathologists for possible aspiration. Care was taken to avoid deleterious effects associated with positioning frail, neurologically compromised patients in a supine or Trendelenburg position for 30 minutes. Patients with feeding tubes had them turned off and flushed at least 1 hour before positioning in supine or Trendelenburg. The research team treated and monitored one patient a day. After chest optimization, patients were repositioned in Fowler's position at $45^{\circ}$ (head-up position).

\section{Experimental Design}

Use of crossover experimental design for this preliminary clinical study enabled a reduction in sample size. Power analysis conducted for SBT duration exceeded 80 percent and indicated that 12 subjects in a crossover design constituted an adequately powered study. Approval to conduct medical research at the Bilirakis SCI Center of Excellence, James A. Haley Veterans Hospital, Tampa, Florida, was obtained from institutional review boards (IRBs) at University of South Florida, Tampa, Florida, and Touro University International, Cypress, California.

Data were collected pre- and post-chest optimization procedures and pre- and post-SBT sessions (Figure 1). Baseline measurements were performed with patients sitting in bed at $45^{\circ}$ in Fowler's position. Mechanical ventilation was provided with a Puritan Bennett 760 mechanical ventilator (Puritan Bennett LLC; Boulder, Colorado). TCO or SCO was randomly administered to each participant. Patients remained within the following clinical parameters before and during implementation of protocol: pulse oximetric saturation $\left(\mathrm{SpO}_{2}\right) \geq 94$ percent on $\mathrm{FIO}_{2}$ of 21 percent, heart rate (HR) $<120$ beats a minute, spontaneous respiratory rate $<30$ breaths a minute, exhaled tidal volume $\geq 400 \mathrm{~mL}$ on synchronous intermittent mandatory ventilation one to two breaths and pressure support ventilation $5 \mathrm{~cm} \mathrm{H}_{2} \mathrm{O}$ pressure (dihydrogen monoxide [water]), body temperature $<101^{\circ} \mathrm{F}$, prealbumin 18 to $45 \mathrm{mg} / \mathrm{dL}$, white blood cell (WBC) 4.2 to $10.3 \mathrm{~g} / \mathrm{L}$, and hemoglobin $>8 \mathrm{mg} / \mathrm{dL}$.

\section{Volumetric Capnography Measurements}

A noninvasive cardiac output (NICO) monitor (Novametrix Medical Systems, Inc; Wallingford, Connecticut) was used for measuring selected cardiopulmonary changes in subjects. Height, weight, and hemoglobin values were used for calibrating measurements for each patient. A CircuVent (DHD Healthcare; Wampsville, New York) circuit was used for bypassing carbon dioxide $\left(\mathrm{CO}_{2}\right)$ /flow and Capnostat $\mathrm{CO}_{2}$ sensors (Respironics; Carlsbad, California) during aerosolization 
of bronchodilators into NICO circuit for preventing decrement in function of sensors. At end-aerosolization, CircuVent device redirected exhaled gas to $\mathrm{CO}_{2}$ /flow and Capnostat $\mathrm{CO}_{2}$ sensors, which allowed resumption of continuous analysis of cardiopulmonary variables.

A NICO monitor uses a partial $\mathrm{CO}_{2}$ rebreathing method to measure and calculate multiple cardiopulmonary variables [19]. The monitor integrates end-tidal $\mathrm{CO}_{2}$ and expiratory flow measurements to generate volumetric capnogram waveforms and numerics. The monitor responds to rapidly changing cardiac output (CO), shunt conditions [20], and various emergency medical conditions [21]. Kallett et al. demonstrated that volumetric capnography accurately calculated physiological dead space in patients with acute respiratory distress syndrome and correlated with standard metabolic cart method [22]. Volumetric capnography is frequently measured intraoperatively and postsurgically for monitoring selected cardiopulmonary variables in patients with reduced mobility and stable metabolism. NICO monitoring of subacute and chronic ventilator-dependent patients has been standard practice in the neurorespiratory ventilator unit at the James A. Haley Veterans Hospital in Tampa, Florida, for several years.

The NICO monitor used for producing volumetric capnograms for this study measured several cardiopulmonary variables, one of which was static compliance, a measure of distensibility of lung and chest wall. The PB 760 mechanical ventilator performs a $2 \mathrm{~s}$ automated breath hold, while the NICO monitor measures gas volume per unit change in pressure. Increased static compliance is associated with decreased work of breathing and prolonged SBT duration.

Therapeutic interventions for supine (control) and Trendelenburg (experimental) conditions consisted of two dichotomous measurement points for chest optimization and two for SBT. At pre-chest optimization, cardiopulmonary measurements were performed with patient on mechanical ventilation in Fowler's position. At postchest optimization, cardiopulmonary measurements were performed 30 minutes after TCO or SCO with patient still in Trendelenburg or supine position and receiving mechanical ventilation. Pre-SBT cardiopulmonary baseline measurements were performed in Fowler's position with the patient on $\mathrm{FIO}_{2}$ of 21 percent gas injection nebulizer (GIN), $5 \mathrm{~cm} \mathrm{H}_{2} \mathrm{O}$ continuous positive airway pressure (CPAP) valve and tracheostomy tube cuff inflated to minimum occluding volume. Post-SBT measurements were performed when either (1) $\mathrm{SpO}_{2}$ decreased to 93 percent on $\mathrm{FIO}_{2}$ of 21 percent any time during SBT session or (2) the patient successfully completed SBT duration of 120 minutes while maintaining $\mathrm{SpO}_{2} \geq 94$ percent on $\mathrm{FIO}_{2}$ of 21 percent. Pre- and post-SBT measurements were performed for measuring differences in selected physiological variables between the beginning and ending of a given SBT.

\section{Chest Optimization}

We calculated CO, alveolar minute volume (MValv), carbon dioxide elimination $\left(\mathrm{VCO}_{2}\right)$ and static chest compliance by averaging three consecutive measurements with patient in Fowler's position before beginning chest optimization. During chest optimization, patients were randomly placed in either the Trendelenburg position $15^{\circ}$ below horizontal or supine position $0^{\circ}$ to horizontal. Although Cameron et al. placed patients with SCI in Trendelenburg at $15^{\circ}$ and $20^{\circ}$ to augment vital capacity (VC) [23], Trendelenburg $15^{\circ}$ was used in the current study largely because of patient preference.

Delivered tidal volume was $15 \mathrm{~mL} / \mathrm{kg}$ of IBW before chest optimization and $20 \mathrm{~mL} / \mathrm{kg}$ of IBW during $30 \mathrm{~min}-$ utes of chest optimization. Clinical researchers increased tidal volume to $20 \mathrm{~mL} / \mathrm{kg}$ by delivering supplemental gas using a small volume nebulizer (SVN) (Teleflex Medical; Research Triangle Park, North Carolina), placed in-line on the proximal inspiratory limb of the ventilator circuit. At the end of chest optimization, patients remained in Trendelenburg or supine position, SVN was turned off and measurements were obtained for comparing physiological performance pre- and post-chest optimization.

During chest optimization, clinical researchers mobilized sputum by simultaneously performing (1) two to three passes of tracheal suctioning with Ballard catheter closed suction system (Kimberly-Clark; Roswell, Georgia), (2) four to five cycles of mechanical insufflation/exsufflation $\pm 40 \mathrm{~cm} \mathrm{H}_{2} \mathrm{O}$ with mechanical insufflator/exsufflator (MIE) (Respironics; Kennesaw, Georgia) on manual mode with $\pm 5 \mathrm{~s}$ inspiratory/expiratory time plus 5 s pause, and (3) a 20 min session of bed-based thoracic vibration with Total Care Sport bed (Hill-Rom; Batesville, Indiana) at $25 \mathrm{~Hz}$ on high intensity. MIE has a very significant role in assisting secretion clearance. At least one study has determined that even though the amount of sputum recovered with and without MIE is similar, the use of MIE shortened the time needed to achieve airway clearance [18]. 
After chest optimization measurements, abdominal binders were applied in the supine position and patients were returned to Fowler's position to begin SBT, scheduled to last 2 hours. MValv and rapid shallow breathing index (RSBI) were measured during SBT. CO was not measured during SBT because reliable measurements are not obtainable unless the patient is assisted by a ventilator. Clinical researchers performed post-SBT measurements when either (1) $\mathrm{SpO}_{2}$ decreased to 93 percent on $\mathrm{FIO}_{2}$ of 21 percent at any time during SBT session or (2) the patient successfully completed SBT duration of 120 minutes while maintaining $\mathrm{SpO}_{2} \geq 94$ percent on $\mathrm{FIO}_{2}$ of 21 percent.

\section{Data Analyses}

Effect of body positioning on SBT was determined by a paired samples $t$-test. Interaction effects and main effects of positioning and time on cardiopulmonary variables were determined by repeated-measures analysis of variance. Statistical analysis was performed with commercially available software, SPSS 17 (SPSS, Inc.; Chicago, Illinois). Differences were considered significant if $p<0.05$.

\section{RESULTS}

Subjects constituted a neurologically heterogeneous group of male veteran patients with low traumatic CSCI (C3-C5) and mean age of 55 years. Non-Active Duty veterans accounted for 83 percent of those studied. Most (75\%) participants were at least 51 years old and presented with age-related disease-specific comorbidities that included bradycardia, atrial fibrillation, diabetes, neuropathic pain, and muscular spasticity. Most (84\%) participants had normal body mass index, while 16 percent were either overweight or obese (Table 1). Subacute and chronic injuries were matched; 50 percent of subjects had been injured $<5$ years and 50 percent for $>5$ years.

All patients exhibited either American Spinal Injury Association (ASIA) A or ASIA B severity of injury (Table 2). All subjects were mechanically ventilated with minimum tidal volume of $1,200 \mathrm{~mL}$ and minimum assistcontrol rate of six breaths a minute. Average tidal volume of $1,200 \mathrm{~mL}$ is an SCI-appropriate tidal volume $(15 \mathrm{~mL} /$ kg IBW) frequently used in neurorespiratory rehabilitation and is greater than tidal volume $(6-7 \mathrm{~mL} / \mathrm{kg})$ employed conventionally in intensive care environments. Patients with CSCI sometimes exhibit a lower blood
Table 1.

Age, duty status, sex, race, and body mass index (BMI) of ventilatordependent patients $(N=12)$ with cervical spinal cord injury.

\begin{tabular}{cclcc}
\hline $\begin{array}{c}\text { Age }^{*} \\
\text { (yr) }\end{array}$ & $\begin{array}{c}\text { Duty } \\
\text { Status }\end{array}$ & Sex & Race & BMI $^{\ddagger}$ \\
\hline 24 & Active & Male & Asian & 21.57 \\
26 & Active & Male & Caucasian & 19.41 \\
74 & Nonactive & Male & Caucasian & 24.41 \\
63 & Nonactive & Male & Caucasian & 21.25 \\
66 & Nonactive & Male & Caucasian & 24.56 \\
32 & Nonactive & Male & Caucasian & 35.66 \\
51 & Nonactive & Male & Caucasian & 18.66 \\
70 & Nonactive & Male & Caucasian & 27.55 \\
63 & Nonactive & Male & Caucasian & 24.53 \\
58 & Nonactive & Male & Caucasian & 24.01 \\
71 & Nonactive & Male & Caucasian & 19.96 \\
62 & Nonactive & Male & Caucasian & 19.94 \\
\hline
\end{tabular}

${ }^{*}$ Age (mean $=55.00 \pm 17.79$ standard deviation).

${ }^{\dagger}$ Duty status refers to official military status of patient. Two patients in study were Active Duty pending honorable discharge.

${ }^{\ddagger}$ Mean BMI $=23.46 \pm 4.69$ standard deviation, with normal BMI $<25$, overweight 25-30, and obese $\geq 30$.

Table 2.

Years post-spinal cord injury (post-SCI), highest level of injury (LOI), severity of injury, and ventilator settings of ventilator-dependent patients $(N=12)$ with cervical SCI.

\begin{tabular}{rcccc}
\hline \multirow{2}{*}{$\begin{array}{c}\text { Post-SCI } \\
(\mathbf{y r})^{*}\end{array}$} & $\begin{array}{c}\text { Highest } \\
\text { LOI }\end{array}$ & $\begin{array}{c}\text { ASIA } \\
\text { Severity of } \\
\text { Injury }\end{array}$ & \multicolumn{2}{c}{$\begin{array}{c}\text { Ventilator } \\
\text { Settings }\end{array}$} \\
\cline { 4 - 5 } 1 & C5 & A $^{\ddagger}$ & 1,200 & 6 \\
1 & C3 & B $^{\S}$ & 1,200 & 6 \\
10 & C4 & B & 1,200 & 6 \\
3 & C4 & A & 1,200 & 6 \\
2 & C5 & B & 1,200 & 6 \\
3 & C3 & B & 1,400 & 10 \\
30 & C4 & A & 1,200 & 6 \\
4 & C4 & A & 1,200 & 6 \\
25 & C5 & B & 1,200 & 6 \\
27 & C5 & B & 1,200 & 6 \\
25 & C4 & B & 1,200 & 6 \\
15 & C5 & B & 1,200 & 6
\end{tabular}

Note: Patients also received fractional-inspired oxygen concentration at $21 \%$ and positive end-expiratory pressure at $5 \mathrm{~cm} \mathrm{H}_{2} \mathrm{O}$ (water).

${ }^{*}$ Mean $=12.17 \pm 11.55$ standard deviation.

${ }^{\dagger}$ Rate $=$ preprogrammed breaths per minute delivered by ventilator on Assist/ Control mode.

${ }^{\ddagger}$ America Spinal Injury Association (ASIA) impairment scale designation for complete injury. No motor or sensory function is preserved in sacral (S) levels S4-S5.

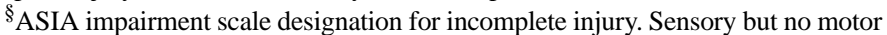
function is preserved below neurological level and includes levels S4-S5.

$\mathrm{C}=$ cervical (number refers to specific level of cervical SCI), VT = tidal volume (15 mL/kg ideal body weight at pre- and post-chest optimization). 
pressure than what is normally encountered in neurologically intact individuals. Subjects did not need to be treated for autonomic dysreflexia, hyperthermia, hypoxia, hypercarbia, and/or hypotension during the study period. Patients had normal prealbumin values, indicating nutritional repletion of skeletal muscles and readiness to perform SBT. C-reactive proteins and WBC values were normal, while hematocrit values were slightly below normal but not below $8 \mathrm{mg} / \mathrm{dL}$.

\section{Prolonged Spontaneous Breathing Trial}

Average SBT duration (mean \pm standard deviation $=$ $87.67 \pm 38.42 \mathrm{~min}$ ) following TCO was significantly ( $p=$ 0.001 ) greater than average SBT duration (mean \pm standard deviation $=33.50 \pm 30.34 \mathrm{~min}$ ) following SCO.

\section{Improvements During Trendelenburg Chest Optimization}

Multivariate analysis revealed TCO was associated with a 20 percent increase $(p<0.001)$ in CO, during which it increased by an average $1.86 \mathrm{~L} / \mathrm{min}$ (Figure 2). TCO was also associated with a-

- 14 percent increase $(p<0.004)$ in MValv, during which it increased by an average 1.13 L (Figure 3).

- 15 percent increase $(p<0.001)$ in $\mathrm{VCO}_{2}$, during which it increased by an average $38.58 \mathrm{~mL} / \mathrm{min}$ (Figure 4).

- 13 percent increase $(p<0.002)$ in Cst, during which it increased by an average $9.92 \mathrm{~mL} / \mathrm{cm} \mathrm{H}_{2} \mathrm{O}$ (Figure 5).

Sputum wet weight measurements pre- and post-TCO and pre- and post-SCO revealed no significant change between time (pre- vs post-) or position (Trendelenburg vs supine).

\section{Improvements During Spontaneous Breathing Trial}

Multivariate analysis revealed that SBT following TCO was associated with a 7.43 percent increase $(p<$ 0.03) in MValv, while SBT following SCO was associated with 13.96 percent decrease in MValv. Average rate of decline in MValv was $0.68 \mathrm{~L} / \mathrm{h}$ during SBT following TCO and 1.6 L/h during SBT following SCO (Figure 6).

Multivariate analysis also revealed that SBT following TCO was associated with a 13 percent increase $(p<$ 0.002) in RSBI, while SBT following SCO was associated with a 33 percent increase in RSBI. Average rate of increase in RSBI was 6 units/h during SBT following TCO and 57 units/h during supine following SCO (Figure 7).

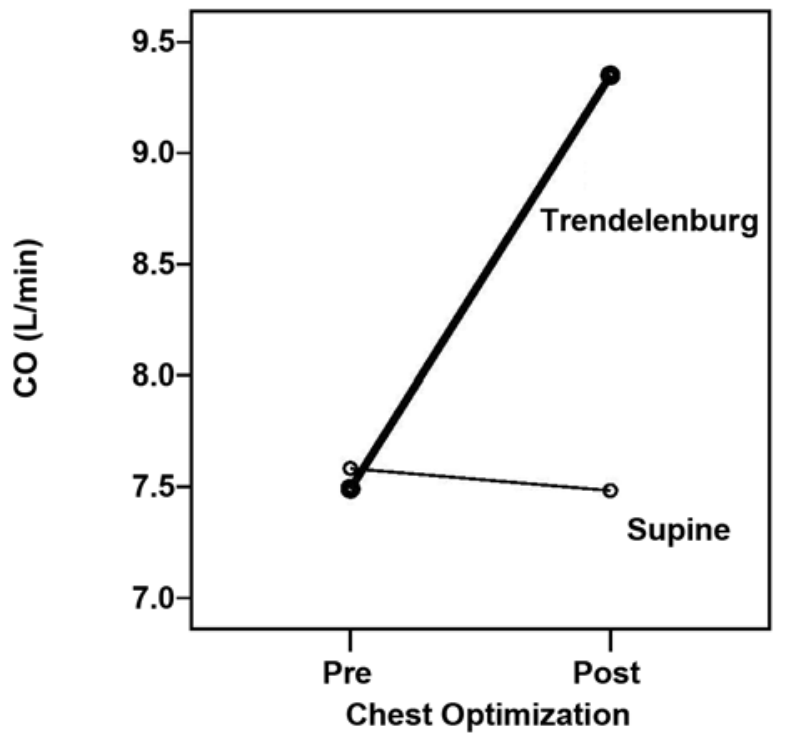

Figure 2.

Measurements of cardiac output (CO) during chest optimizations (Trendelenburg and supine). Trendelenburg increased CO 20\%, an average of $1.86 \mathrm{~L} / \mathrm{min}$.

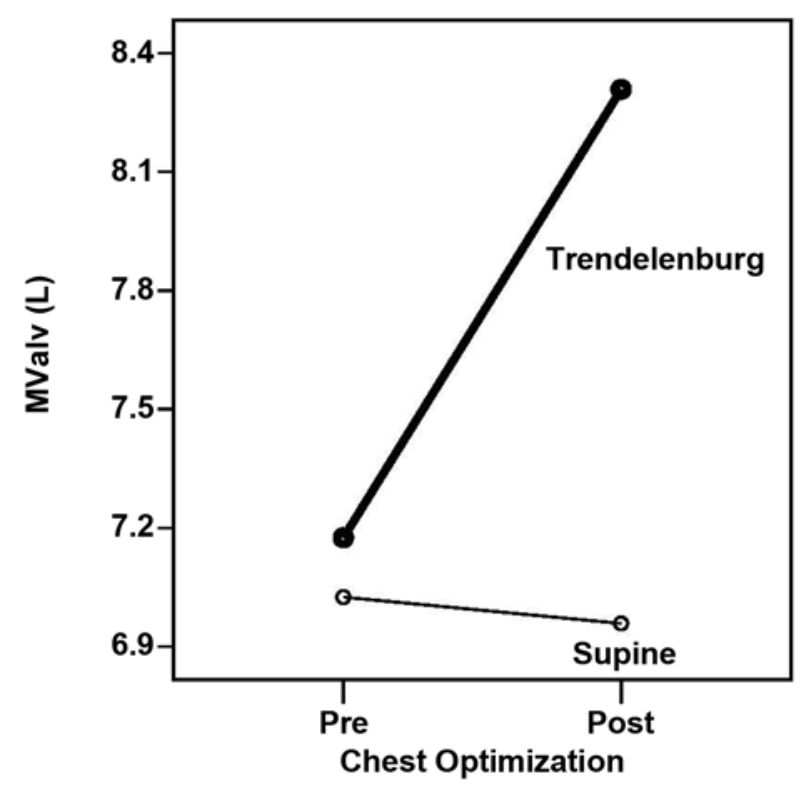

Figure 3.

Measurements of alveolar minute volume (MValv) during chest optimizations (Trendelenburg and supine). Trendelenburg increased MValv $14 \%$, an average of $1.13 \mathrm{~L}$. 


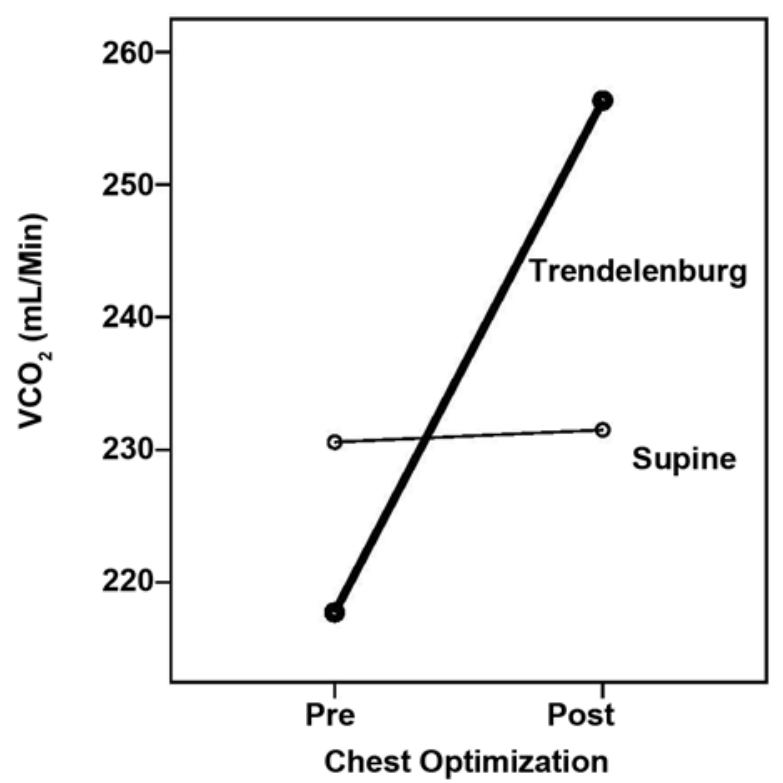

Figure 4.

Measurements of carbon dioxide elimination $\left(\mathrm{VCO}_{2}\right)$ during chest optimizations (Trendelenburg and supine). Trendelenburg increased $\mathrm{VCO}_{2} 15 \%$, an average of $38.58 \mathrm{~mL} / \mathrm{min}$.

\section{DISCUSSION}

Findings of the present study revealed that ventilatordependent patients who were clinically prepared with TCO performed an SBT that lasted an average 53 minutes longer than those clinically prepared with SCO.

\section{Recumbent Body Positioning}

The role of body positioning during multimodal chest physiotherapy in patients with low CSCI has not been adequately studied. Although the scientific literature has addressed a spectrum of issues pertaining to CSCI, few studies have generated high-level clinical research findings that may be used to design neurorespiratory rehabilitation and ventilator weaning protocols for ventilatordependent patients with low tetraplegia. While lack of scientific evidence does not necessarily imply lack of benefit [24], part of the difficulty in achieving meaningful research findings may be that chest optimization techniques are studied in a manner similar to which they are clinically implemented, one at a time. Studying multimodal chest physiotherapy as a bundle and varying one modality at a time for determining its relative contribution to the bundle is a strategy that may provide greater insight into the efficacy of the intervention. In addition, a multi-

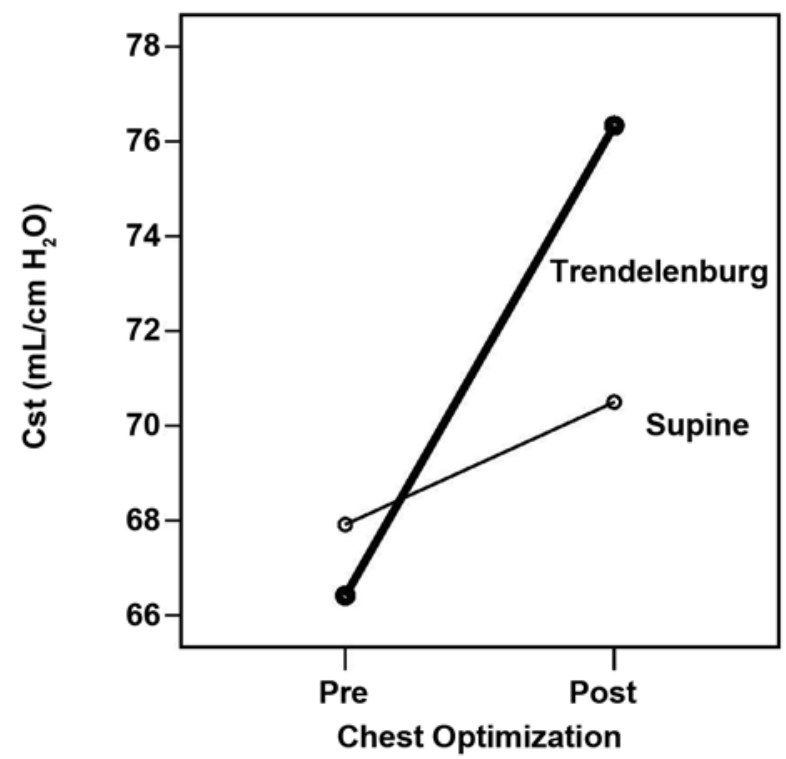

Figure 5.

Measurements of static chest compliance (Cst) during chest optimizations (Trendelenburg and supine). Trendelenburg increased Cst 13\%, an average of $9.92 \mathrm{~mL} / \mathrm{cm} \mathrm{H}_{2} \mathrm{O}$ pressure.

modal clinical approach may enable individual patients to benefit from the combined and individual relative strengths of modes that comprise the clinical bundle [25].

In 2005, the Consortium for Spinal Cord Medicine (CSCM) established best practices by publishing evidence-based guidelines for patients with low SCI [26]. One CSCM recommendation was to consider placing mechanically ventilated patients with SCI in either supine or Trendelenburg position for improving ventilation. This recommendation was based on studies by Forner et al. who noted that patients with low CSCI exhibited average forced VC that was $300 \mathrm{~mL}$ greater in these positions than in sitting position [27].

Early studies by Cameron et al. measured VC of patients with low CSCI in three body positions: $15^{\circ}$ headdown position (Trendelenburg), $15^{\circ}$ head-up position (Fowler's), and supine [23]. The effect of body positioning on VC measurements of these patients was not only opposite to what is typically observed in patients without CSCI but is also more pronounced and consistent. Compared with baseline VC measurements during supine body positioning, findings revealed a 7 percent decrease with Fowler's positioning versus a 6 percent increase with Trendelenburg positioning. The placement of a lone patient in a $20^{\circ}$ head-down position resulted in a 10 percent 


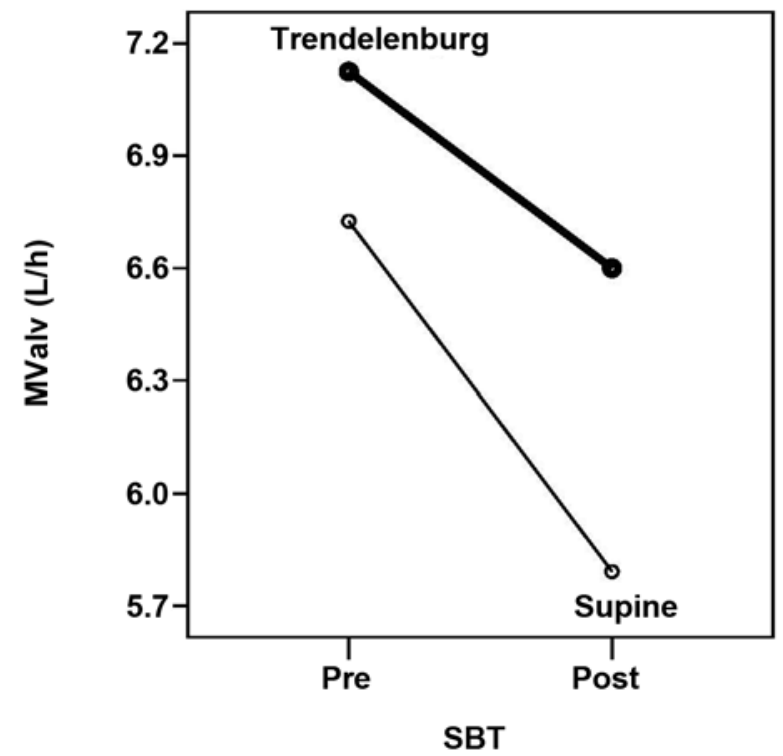

Figure 6.

Alveolar minute volume (MValv) during spontaneous breathing trial (SBT) pre- and postchest optimizations. MValv average rate of decline was $0.68 \mathrm{~L} / \mathrm{h}$ during SBT following Trendelenburg and $1.6 \mathrm{~L} / \mathrm{h}$ following supine.

increase in VC. Hence, increases in VC in those with low tetraplegia may be directly related to degree of body positioning below horizontal and inversely related to degree of body positioning above horizontal. To determine whether the increased VC was transient, Cameron et al. conducted an $n$-of- 1 study, in which a patient with tetraplegia was maintained in Trendelenburg for 4 hours, during which VC remained unchanged [23].

When a person is in the sitting position, abdominal contents push the diaphragm cephalad, placing it at a mechanical disadvantage $[4,13,23]$. The reduction in VC and associated dyspnea experienced when a patient with low tetraplegia sits upright can be improved with an abdominal binder. This device reconfigures the shape of the diaphragm so that it resembles a parachute or dome. The reconfiguration increases tidal volume and improves efficiency of the ventilatory mechanics [28] by increasing the zone of apposition of the diaphragm relative to the caudal circumference of the rib cage [29]. The domed diaphragm lifts the lower edges of the rib cage by using the intestines as a fulcrum. Abdominal binding influences the rib cage motion over the entire range of inspiratory capacity of patients with CSCI [29].

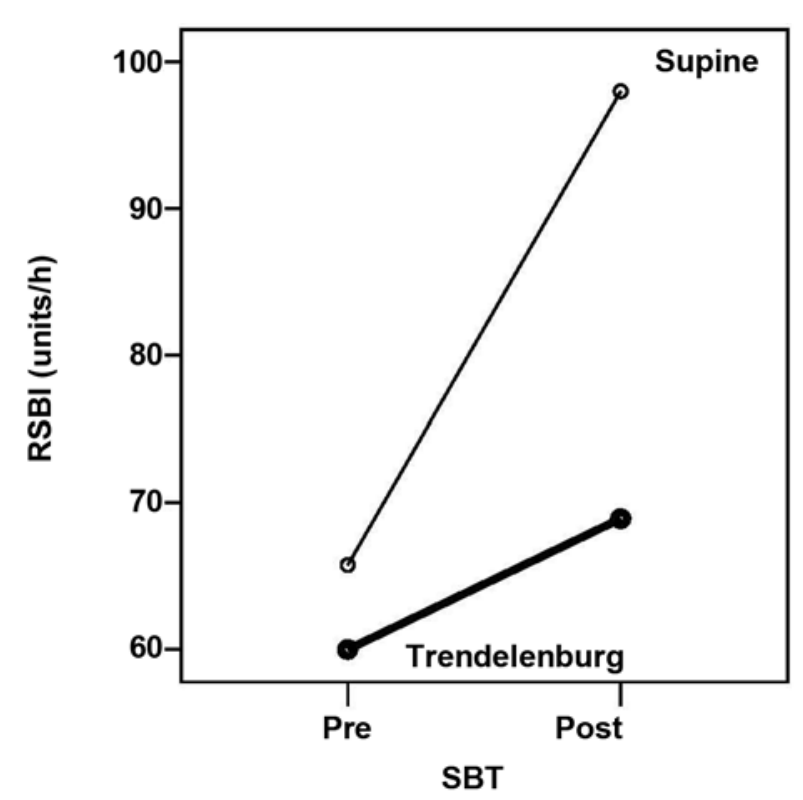

Figure 7.

Rapid shallow breathing index (RSBI) during spontaneous breathing trial (SBT). Trendelenburg increased SBT 33\%, in RSBI.

Findings of the current study are consistent with the work of Cameron et al. [23], as well as the recent work by Brown et al. [30], who suggested that application of an abdominal binder to patients with SCI similarly domed the diaphragm and improved mechanical efficiency of subsequent diaphragmatic contractions during inspiration. Whether the doming of the diaphragm is caused by body positioning or by abdominal binding, an improvement is apparent in mechanical efficiency of the rib cage of patients with CSCI that may not be clinically apparent in controls without CSCI.

\section{Trendelenburg Chest Optimization}

Patients who received TCO completed 73 percent of 120 minutes allotted for an SBT session, while subjects who received SCO completed 28 percent. TCO was associated with significant increase in $\mathrm{CO}$, which was unexpected given the documented tendency of patients with CSCI to exhibit attenuated hemodynamic responses. In controls without CSCI, blood flow during exercise is proportional to CO [30]. Takahashi et al. measured the HR of patients with CSCI at beginning of exercise and found it less than the HR in controls without CSCI [31]. However, at the end of exercise, HR was similar in both groups, suggesting it increases very slowly in patients 
with CSCI, possibly due to decelerating effects of parasympathetic predominance [32]. Exercise-mediated increases in $\mathrm{CO}$ have been attributed primarily to increased stroke volume [33] rather than increased rate in those with CSCI.

Increased CO would be expected to increase blood flow to diaphragm and enhance ability of patients with CSCI to perform extended SBT sessions. The primary rationale for placing ventilator-dependent patients with CSCI in Trendelenburg has been to mobilize sputum and adequately expand alveoli in lung regions vulnerable to atelectasis [34]. Positioning in Trendelenburg to increase CO systematically has not traditionally been a specific clinical objective. Nevertheless, current findings suggest that Trendelenburg positioning may be associated with an increased CO that may last as much as 120 minutes in some patients. Increasing blood flow to ventilatory muscles immediately before exercise is particularly important because some patients with chronic SCI have femoral artery diameters and maximal blood flows that are 50 percent of controls without SCI [35]. After CSCI, pathological adaptations in peripheral circulation and skeletal muscles decrease capacity for exercise [36]. Circulatory impairments in those with CSCI suggest skeletal muscle fatigue is much more likely to occur than in controls without SCI. Hence, the ability to perform extended SBT sessions may depend on augmented blood flow following TCO.

During TCO, MValv increased 14 percent, $\mathrm{VCO}_{2}$ increased 15 percent, and Cst increased 13 percent. Finding that improvements in MValv and $\mathrm{VCO}_{2}$ were associated with increased Cst supports the hypothesis that positioning in Trendelenburg during chest optimization augments alveolar recruitment [37]. During TCO, a shift in pulmonary blood volume from basilar to apical lung regions would be expected to reduce hydrostatic pressure surrounding basilar alveoli, thus enabling them to accommodate a greater volume of gas, as shown by increases in each of the aforementioned cardiopulmonary variables. Trendelenburg positioning would be expected to accentuate transpulmonary pressures in basilar lung regions by alveolar hyperinflation promoting alveolar recruitment, reversing atelectasis, and increasing surfactant production. Stretching pneumocyte type II cells in basilar alveoli is associated with delayed increase in surfactant in those alveoli. Therefore, hyperinflation of basilar alveoli reduces the tendency to develop atelectasis, not only because alveoli expand mechanically but also because surfactant reduces alveolar surface tension and mitigates atelectasis [38].

\section{Spontaneous Breathing Trial}

In patients without CSCI, the ability to perform an adequate SBT is an important indicator of adequate ventilatory function and may predict clinical readiness to begin progressive ventilator-free breathing sessions that often precede discontinuation from mechanical ventilation. Transfer from the mechanical circuit of ventilator to mechanical circuit of the GIN/CPAP device was performed in $<3 \mathrm{~s}$ so that the SBT session could begin. Use of the GIN/CPAP device instead of commonly used devices, such as the standard aerosol tracheostomy collar or T-piece without CPAP valve, is preferred, given the ability of GIN/CPAP to enhance functional residual capacity (FRC). A recent study of 60 patients without CSCI in a medical intensive care unit revealed that RSBI was significantly lower when patients were placed on $5 \mathrm{~cm} \mathrm{H}_{2} \mathrm{O}$ CPAP versus T-piece [39]. An RSBI value $\leq 105$ breaths/min/L predicts a successful attempt at weaning, while an RSBI $\geq 105$ breaths/min/L predicts an unsuccessful attempt at weaning.

Findings of the current study revealed a significant difference in MValv for SBT following TCO. Even though application of the GIN/CPAP device increases FRC of lung, dilates airways, and mechanically splints chest wall, it does not provide active, intermittent lung expansion characteristic of mechanical ventilation. Nevertheless, our experience suggests that GIN/CPAP may improve ventilatory capacity and/or reduce ventilatory workload during SBT sessions in some patients with CSCI. During SBT, the average rate of decline in MValv was $0.68 \mathrm{~L} / \mathrm{h}$ for subjects who underwent TCO and $1.6 \mathrm{~L} / \mathrm{h}$ for those who underwent SCO. Hence, augmenting CO, MValv, $\mathrm{VCO}_{2}$, and Cst during TCO before undertaking SBT appears associated with a reduced rate of decline in MValv during SBT.

A significant difference in RSBI occurred between SBT following TCO versus SBT following SCO. The increase in RSBI during SBT following SCO was 57 units/h, while the increase during SBT following TCO was 6 units/h. In addition, the rate of increase in the index during SBT following SCO was significantly greater than the rate of increase during SBT following TCO. While we expected a greater increase in RSBI during SBT following SBO, we did not anticipate the increased rate of change. RSBI has traditionally been used in intensive care units as an 
important indicator of ventilatory capacity in mechanically ventilated patients without CSCI who are candidates for weaning from the ventilator [40]. While many such clinical indicators have been proposed, RSBI as proposed by Yang and Tobin has survived as the only index experimentally determined to be reliable and valid [41]. In the current study, RSBI was measured in real time, as recommended by Haryadi et al. [42], rather than intermittently.

Increases in RSBI (maximum 105) reflect a progressive decline in ventilatory capacity as SBT proceeds. The significant (33\%) increase in RSBI during SBT following SCO suggests that patients who underwent SCO may not have received a significant physiological enhancement and were not able to perform an extended SBT. The smaller (13\%) increase in RSBI during SBT following TCO suggests patients who underwent TCO received a significant physiological enhancement and were able to perform an extended SBT. The greater rate of increase in RSBI for patients who underwent SBT following SCO was particularly revealing because it suggests that without the physiological enhancement in CO, MValv, $\mathrm{VCO}_{2}$, and Cst provided by TCO, a relatively rapid decline in SBT duration occurs. Future research will be required to determine the degree to which increases in CO during TCO persist during subsequent SBT sessions.

Trendelenburg positioning may be the recumbent body position of choice during chest optimization for improving selected cardiopulmonary variables immediately before undertaking SBTs that lead to progressive ventilator-free breathing and liberation from mechanical ventilation. Trendelenburg body positioning may have enhanced chest mechanics during the chest optimization preparatory phase, while abdominal binding instituted during a SBT may have worked synergistically to improve and maintain mechanical efficiency of the diaphragm. These complementary maneuvers might explain changes in CO, MValv, $\mathrm{VCO}_{2}$, and Cst during TCO and in MValv and RSBI during SBT. An n-of- 1 study is recommended for clinicians attempting to determine empirically whether application of an abdominal binder, following TCO, extends subsequent SBT duration for a given patient with CSCI attempting to wean from mechanical ventilation.

\section{Limitations}

Limitations of this preliminary study include the use of short-term outcomes following a single treatment session as described by Hess [24] and neurological hetero- geneity. Sample heterogeneity is a common dilemma in pilot studies involving patients with CSCI and is one reason why multicenter studies consisting of similarly injured patients are undertaken. Employment of patients as their own controls as occurred in the current study may have mitigated some effects of neurological heterogeneity. SBT duration is an end point that provides useful information of interest to clinicians planning long-term studies of this clinically fragile, historically underserved cohort. Long-term effects on pulmonary functional outcomes arising from routine administration of TCO were not addressed but should be investigated in future longitudinal studies. Long-term indicators of particular interest include changes in endurance and strength, ventilatory mechanics, metabolism, functional outcomes, pulmonary function, cardiothoracic imaging, days in hospital, days in therapy, frequency of cardiopulmonary exacerbations, days at home, length of life, and healthrelated quality of life [43].

\section{CONCLUSIONS}

Clinical progress in weaning patients from mechanical ventilation may be associated with the ability to progressively increase the length of time a ventilatordependent patient with CSCI can perform an SBT. The current study suggests TCO is associated with a significant increase in SBT duration. Future research should focus on further characterizing physiological mechanisms by which TCO prolongs SBTs. This information should enable clinicians to design and implement safer and more effective, evidence-based protocol-guided rehabilitation and weaning strategies for ventilator-dependent patients with low CSCI.

\section{ACKNOWLEDGMENTS}

\section{Author Contributions:}

Study concept and design: C. J. Gutierrez, M. Tanasescu, G. Curtis. Acquisition of data: C. J. Gutierrez, C. Stevens.

Analysis and interpretation of data: C. J. Gutierrez, C. Stevens

C. Pope, G. Curtis.

Statistical Analysis: C. J. Gutierrez, M. Tanasescu, G. Curtis.

Drafting of manuscript: C. J. Gutierrez.

Critical revision of manuscript for important intellectual content:

C. J. Gutierrez, J. Merritt, C. Pope, M. Tanasescu.

Administrative, technical, or material support: C. Stevens, J. Merritt, C. Pope, M. Tanasescu, G. Curtis. 
Study supervision: C. Stevens, J. Merritt, C. Pope, M. Tanasescu. Financial Disclosures: The authors have declared that no competing interests exist.

Funding/Support: This material was unfunded at the time of manuscript preparation.

Institutional Review: Findings are based on IRB-approved clinical research supported by VA, James A. Haley Veterans Hospital, Bilirakis Spinal Cord Injury Center of Excellence, Neurorespiratory Care Program.

Participant Follow-Up: The authors plan to inform participants of the publication of this article.

\section{REFERENCES}

1. Lemons VR, Wagner FC Jr. Respiratory complications after cervical spinal cord injury. Spine. 1994;19(20):2315-20. [PMID: 7846577]

2. Jackson AB, Groomers TE. Incidence of respiratory complications following spinal cord injury. Arch Phys Med Rehabil. 1994;75(3):270-75. [PMID: 8129577] DOI:10.1016/0003-9993(94)90027-2

3. Lanig IS, Peterson WP. The respiratory system in spinal cord injury. Phys Med Rehabil Clin N Am. 2000;11(1):29-43. [PMID: 10680156]

4. Winslow C, Rozovsky J. Effect of spinal cord injury on the respiratory system. Am J Phys Med Rehabil. 2003;82(10): 803-14. [PMID: 14508412] DOI:10.1097/01.PHM.0000078184.08835.01

5. Ledsome JR, Sharp JM. Pulmonary function in acute cervical spinal cord injury. Am Rev Respir Dis. 1981;124(1): 41-44. [PMID: 7258818]

6. Ball PA. Critical care of spinal cord injury. Spine. 2001; 26(24 Suppl):S27-S30. [PMID: 11805605]

DOI:10.1097/00007632-200112151-00006

7. Braverman J, Ehlen J. Cervical spinal cord injury: The role of high frequency chest compression therapy in the management of respiratory complications. Respir Ther. 2009;4(5): 27-31.

8. Carter RE. Respiratory aspects of spinal cord injury management. Paraplegia. 1987;25(3):262-66. [PMID: 3601438]

9. Bellamy R, Pitts FW, Stauffer ES. Respiratory complications in traumatic quadriplegia. Analysis of 20 years' experience. J Neurosurg. 1973;39(5):596-600. [PMID: 4743566] DOI:10.3171/jns.1973.39.5.0596

10. Kiwerski J. Respiratory problems in patients with high lesion quadriplegia. Int J Rehabil Res. 1992;15(1):49-52. [PMID: 1601568]

11. Claxton AR, Wong DT, Chung F, Fehlings MG. Predictors of hospital mortality and mechanical ventilation in patients with cervical spinal cord injury. Can J Anaesth. 1998;45(2): 144-49. [PMID: 9512849]

DOI:10.1007/BF03013253
12. Wallbom A, Naran B, Thomas E. Acute ventilator management and weaning in individuals with high tetraplegia. Top Spinal Cord Inj Rehabil. 2005;10(3):1-7. DOI:10.1310/K4Y4-YDXQ-9VNY-F562

13. Slack RS, Shucart W. Respiratory dysfunction associated with traumatic injury to the central nervous system. Clin Chest Med. 1994;15(4):739-49. [PMID: 7867288]

14. Kacmarek RM, Durbin CG, Barnes TA, Kageler WV, Walton JR, O'Neil EH. Creating a vision for respiratory care in 2015 and beyond. Respir Care. 2009;54(3):375-89. [PMID: 19245732]

15. Gutierrez CJ, Harrow J, Haines F. Using an evidence-based protocol to guide rehabilitation and weaning of ventilatordependent cervical spinal cord injury patients. J Rehabil Res Dev. 2003;40(5 Suppl 2):99-110. [PMID: 15074456] DOI:10.1682/JRRD.2003.10.0099

16. Gutierrez CJ, Haines F. Chest-optimization for patients with SCI at risk for acute ventilatory failure. J Spinal Cord Med. 2004;27(4):422.

17. Dean E. Oxygen transport: a physiologically-based conceptual framework for the practice of cardiopulmonary physiotherapy. Physiotherapy. 1994;80(6):347-56.

18. Chatwin M, Simonds AK. The addition of mechanical insufflation/exsufflation shortens airway-clearance sessions in neuromuscular patients with chest infection. Respir Care. 2009;54(11):1473-79. [PMID: 19863831]

19. Jaffe M. Partial $\mathrm{CO}_{2}$ rebreathing cardiac output-Operating principles of the NICO system. J Clin Monit Comp. 1999; 15(6):387-401. [PMID: 12578034$]$ DOI:10.1023/A:1009981313076

20. Orr J, Kofoed S, Westenskow D. Evaluation of partial rebreathing cardiac output measurement in animals. Crit Care Med. 1999;27(1):A114. DOI:10.1097/00003246-199901001-00300

21. Maxwell L, Ellis E. Secretion clearance by manual hyperinflation: Possible mechanisms. Physiother Theory Pract. 1998;14(4):189-97. DOI:10.3109/09593989809057165

22. Kallet R, Daniel BM, Garcia O, Matthay M. Accuracy of physiologic dead space measurements in patients with acute respiratory distress syndrome using volumetric capnography: Comparison with the metabolic monitor method. Respir Care. 2005;50(4):462-67. [PMID: 15807908$]$

23. Cameron GS, Scott JW, Jousse AT, Botterell EH. Diaphragmatic respiration in the quadriplegic patient and the effects of position on his vital capacity. Ann Surg. 1955;141(4): 451-56. [PMID: 14362375]

DOI:10.1097/00000658-195504000-00004

24. Hess DR. The evidence for secretion clearance techniques. Respir Care. 2001;46(11):1276-93. [PMID: 11679147]

25. Lester MK, Flume PA. Airway-clearance therapy guidelines and implementation. Respir Care. 2009;54(6):733-53. [PMID: 19467161] 
26. Consortium for Spinal Cord Medicine. Respiratory management following spinal cord injury: A clinical practice guideline for health-care professionals. J Spinal Cord Med. 2005;28(3):259-93. [PMID: 16048145]

27. Forner JV, Llombart RL, Valledor MC. The flow-volume loop in tetraplegics. Paraplegia. 1977;15(3):245-51. [PMID: 593715]

28. Gilbert J. Critical care management of the patient with acute spinal cord injury. Crit Care Clin. 1987;3(3):549-67. [PMID: 3332214]

29. Olive JL, McCully KK, Dudley GA. Blood flow response in individuals with incomplete spinal cord injuries. Spinal Cord. 2002;40(12):639-45. [PMID: 12483497] DOI:10.1038/sj.sc.3101379

30. Brown R, DiMarco AF, Hoit JD, Garshick E. Respiratory dysfunction and management in spinal cord injury. Respir Care. 2006;51(8):853-70. [PMID: 16867197]

31. Takahashi M, Sakaguchi A, Matsukawa K, Komine H, Kawaguchi K, Onari K. Cardiovascular control during voluntary static exercise in humans with tetraplegia. J Appl Physiol. 2004;97(6):2077-82. [PMID: 15310746] DOI:10.1152/japplphysiol.00546.2004

32. Bhashkar KR, Brown R, O’Sullivan DD, Melia S, Duggan M, Reid L. Bronchial mucus hypersecretion in acute quadriplegia. Macromolecular yields and glycoconjugate composition. Am Rev Respir Dis. 1991;143(3):640-48. [PMID: 2001077]

33. Dela F, Mohr T, Jensen CM, Haahr HL, Secher NH, Biering-Sørensen F, Kjaer M. Cardiovascular control during exercise: Insights from spinal cord-injured humans. Circulation. 2003;107(16):2127-33. [PMID: 12695298]

DOI:10.1161/01.CIR.0000065225.18093.E4

34. Milic-Emili J, Henderson JA, Dolovich MB, Trop D, Kaneko K. Regional distribution of inspired gas in the lung. J Appl Physiol. 1966;21(3):749-59. [PMID: 5912744]

35. Nash MS, Montalvo BM, Applegate B. Lower extremity blood flow and responses to occlusion ischemia differ in experience-trained and sedentary tetraplegic persons. Arch Phys Med Rehabil. 1996;77(12):1260-65. [PMID: 8976309] DOI:10.1016/S0003-9993(96)90190-2
36. Villa E, Gonzalez-Albarrán O, Rábano A, García-Robles R. Effects of hyperinsulinemia on vascular blood flows in experimental obesity. J Steroid Biochem Mol Biol. 1999; 69(1-6):273-79. [PMID: 10419002]

37. Massaro G, Massaro D. Morphologic evidence that large inflations of the lung stimulate secretion of surfactant. Am Rev Respir Dis. 1983;127(2):235-36. [PMID: 6687518]

38. Badet M, Bayle F, Richard J, Guérin C. Comparison of optimal positive end-expiratory pressure and recruitment maneuvers during lung-protective mechanical ventilation in patients with acute lung injury/acute respiratory distress syndrome. Respir Care. 2009;54(7):847-54. [PMID: 19558735]

39. Patel KN, Ganatra KD, Bates JH, Young MP. Variation in the rapid shallow breathing index associated with common measurement techniques and conditions. Respir Care. 2009; 54(11):1449-50.

40. Cohen JD, Shapiro M, Grozovski E, Singer P. Automatic tube compensation-assisted respiratory rate to tidal volume ratio improves prediction of weaning outcome. Chest. 2002; 122(3):980-84. [PMID: 12226043] DOI:10.1378/chest.122.3.980

41. Yang KL, Tobin MJ. A prospective study of indexes predicting the outcome of trials of weaning from mechanical ventilation. N Engl J Med. 1991;324(21):1430-50. [PMID: 2023603]

42. Haryadi DG, Orr JA, Kuck K, McJames S, Westenskow DR. Partial CO2 rebreathing indirect Fick technique for non-invasive measurement of cardiac output. J Clin Monit Comput. 2000;16(5-6):361-74. [PMID: 12580219] DOI:10.1023/A:1011403717822

43. Jain NB, Sullivan M, Kazis LE, Tun CG, Garshick E. Factors associated with health-related quality of life in chronic spinal cord injury. Am J Phys Med Rehabil. 2007;86(5): 387-96. [PMID: 17449983] DOI:10.1097/PHM.0b013e31804a7d00

Submitted for publication August 24, 2009. Accepted in revised form January 11, 2010. 